\title{
La symétrie et ses doubles : approches géographiques
}

\section{Antoine Beyer}

\section{(2) OpenEdition}

\section{Journals}

Édition électronique

URL : http://journals.openedition.org/rge/91

DOI : $10.4000 /$ rge. 91

ISSN : 2108-6478

Éditeur

Association des géographes de l'Est

\section{Édition imprimée}

Date de publication : 1 avril 2007

Pagination : $77-78$

ISSN : 0035-3213

\section{Référence électronique}

Antoine Beyer, «La symétrie et ses doubles : approches géographiques », Revue Géographique de l'Est [En ligne], vol. 47 / 2 | 2007, mis en ligne le 01 janvier 2009, consulté le 24 septembre 2020. URL : http://journals.openedition.org/rge/91 ; DOl : https://doi.org/10.4000/rge.91

Ce document a été généré automatiquement le 24 septembre 2020.

Tous droits réservés 


\title{
La symétrie et ses doubles : approches géographiques
}

\author{
Antoine Beyer
}

1 Concept fréquemment étudié dans d'autres domaines scientifiques de la physique, chimie, biologie ou encore des mathématiques, la symétrie semble encore peu intéresser les géographes. Elle alimente pourtant un riche débat disciplinaire où les sciences humaines ne sont pas en reste. Faut-il en conclure que cette configuration semble intervenir plus rarement aux plus petites échelles qui sont celles de la géographie ? Pourtant la répartition de certains phénomènes à l'échelle du globe, qu'ils soient topographiques, climatiques ou urbains, ne répondent-ils pas à un tel principe ? Peut-être n'en trouvons-nous pas plus souvent l'expression que parce que trop peu d'attention lui est accordée?

2 En réalité, c'est sans doute l'inverse qu'il faut envisager. Le concept de symétrie était omniprésent chez les géographes grecs qui ont fondé notre discipline. La symétrie était alors postulée comme élément régulateur dans la recherche d'un ordre du monde. Pour la pensée géographique antique, la symétrie occupait une place importante, successivement invoquée dans le découpage des zones et des climats du globe, dans la distribution des continents entre hémisphères et jusqu'à rendre compte du cours du Nil ou de la Méditerranée qui partageait les terres émergées pour Hérodote. Au-delà de ses limites évidentes de la connaissance, le principe de symétrie a fonctionné comme un canevas d'intelligibilité globale du monde. Il a conduit à des déductions qui sont pour Jean Bergevin « un bon exemple de la rencontre entre l'inventaire et l'invention ».

3 Si tous les géographes de l'Antiquité n'ont pas porté la même attention à la question de la symétrie, la notion a sans conteste ainsi occupé un rôle central pour nombre de constructions et de représentations du monde. Restée très largement dans l'impensé des théories antiques, elle apparaît comme une notion clé, qui permettait d'articuler des discours d'ordres différents : la géométrie céleste, l'harmonie et l'équilibre des parties au tout, la projection sur le globe du système des valeurs qui rende compte de la diversité de l'humanité. Même recouverte de légendes fabuleuses, les interrogations qu'elle a suscitées, ont inspiré les savants et les explorateurs de la Renaissance et 
alimenté les débats scientifiques pour plusieurs siècles comme l'atteste la recherche au XVIII siècle encore de la "Terra Australis » aux Antipodes comme l'a montré Numa Broc. Aussi, malgré ses limites évidentes, la question de la symétrie a ainsi porté une indéniable fécondité heuristique en géographie. Mal dégagée de la pensée analogique et rarement explicité, le recours à la symétrie semble néanmoins plus proposer une construction spéculative, une lecture possible du monde que la simple description d'une réalité établie. Son étoile pâlit, jusqu'à récemment, avec l'avènement de la science moderne.

Dans cette perspective historique, il est important de rappeler que le terme de " symétrie " désigne pour les Grecs plus qu'une simple figure géométrique, une « juste proportion » fondée sur l'harmonie et l'équilibre (du préfixe sun, « avec » dans l'espace ou le temps et metron, «mesure »). Viollet-le-Duc a bien souligné l'évolution moderne de son acception à partir du XVIII ${ }^{e}$ siècle : "Symétrie veut dire aujourd'hui, dans le langage des architectes, non pas une pondération, un rapport harmonieux des parties d'un tout, mais une similitude des parties opposées ». La notion moderne a donc pris un sens plus restrictif, qui se quantifie pour désigner une composition divisible en parties semblables par rapport à un point, une droite ou un plan, là où la pensée grecque comme l'ancien français associent dans le même terme valeur et mesure ${ }^{1}$. Il y a bien une rupture introduite à la Renaissance qui dévalue l'approche spéculative au profit de mesures et d'observations empiriques. La symétrie comme hypothèse régulatrice disparaît alors du discours scientifique, au profit de ses opposés : l'asymétrie ou la dissymétrie. Les formes territoriales qui jouent du redoublement sont alors soumises à une sévère critique des apparences, souvent présentées sous une forme affaiblie ou par le rejet d'une analogie trompeuse. La notion de symétrie s'exprime alors par son manque : fausses symétries (apparence trompeuse), asymétrie (absence), dissymétrie (défaut), la chiralité (deux objets similaires mais non superposables dans l'espace, car inversés). A partir des années 1960-70, l'analyse spatiale nous a appris à reconnaître des régularités géométriques dans l'hétérogénéité de l'espace géographique, mais fait peu de cas de la symétrie qui aurait pu apparaître comme l'expression la plus évidente. Il est ainsi révélateur que Roger Brunet ait retenu dans les formes élémentaires de son tableau des chorèmes, non la symétrie, c'est-à-dire l'expression positive du phénomène, mais son envers, la dissymétrie.

On assisterait pourtant aujourd'hui à un retour en grâce de cette notion qui semble occuper un rôle unificateur dans les sciences. Que faut-il alors entendre dans les nouvelles acceptions du terme? A partir du Petit Robert, on retiendra de la symétrie une définition restrictive " comme une distribution de parties semblables de part et d'autres d'un axe ou d'un plan ». Ce lien spatial entre les deux éléments ajoute à la simple répétition ou duplication un certain rapport qui en fait un système spatial élémentaire. La symétrie implique, au-delà de la ressemblance ou de la duplication, un principe de position définissant une régularité, voire une interdépendance spatiale. Cette première approche introduit une réflexion sur les formes spéculaires des territoires, prémisses à partir desquels les auteurs des textes ont livré leurs contributions.

6 Ce n'est d'ailleurs pas un hasard si la majorité des articles rencontre des formes symétriques de part et d'autre des frontières, comme si les limites territoriales d'un Etat et ses déplacements avaient une faculté de duplication et de création de symétries. Elles traduisent en fait la recherche spontanée ou volontaire de contrepoints et 
d'équilibres politiques aussi bien dans les formes matérielles que dans la confrontation symbolique entre puissances. C'est bien par cette dynamique frontalière que la notion de symétrie est appelée à croiser l'espace couvert par la RGE, celui de l'Europe centrale et orientale.

7 La symétrie peut d'abord être spontanément suggérée par des données naturelles. Ainsi, la topographie du fossé rhénan ouvre des perspectives non seulement de comparaison entre les aménagements des versants alsaciens et badois, mais constitue surtout l'opportunité de transfert de données appliquées au ruissellement et à la pollution des nappes phréatiques par les pesticides. Pour Caroline Grégoire, Jens Lange, Agnès Herrmann, la référence à la symétrie a ici une fonction heuristique qui conduit au transfert et à l'ajustement de modèle.

8 Au-delà d'une donnée statique, la symétrie est fille des flux, de la circulation ou de son empêchement. Raymond Woessner montre comment le territoire symétrique de Mulhouse-Montbéliard (TS2M) s'est historiquement constitué en système industriel et urbain dual. L'axe de partage qui était initialement faible et où la topographie a pu jouer le rôle d'un prétexte, s'est renforcé et structuré par l'établissement des limites politiques fortes entre États, puis entre unités administratives françaises (Régions et départements). Axe de partage, la ligne frontière trouve aujourd'hui, à mi-chemin entre les deux pôles automobiles une attractivité renouvelée, comme peut en témoigner le développement de l'Aéroparc de Fontaine dont l'accueil d'activités industrielles et logistiques s'est subsisté à sa vocation première. L'analyse ainsi formalisée pourrait certainement s'appliquer avec succès au bipôle lorrain de la métropole Metz / Nancy dont profite le point médian qui est Pont-à-Mousson.

La dynamique temporelle est au cœur des considérations développées par Franck Tétart à travers l'analyse de la situation d'une symétrie redoublée. L'enclave de Koenigsberg/Kaliningrad (même si les contours des territoires considérés ne coïncident pas dans le détail, du fait du rattachement de la partie méridionale de la Prusse Orientale à la Pologne), offre l'exemple original d'une position d'enclavement répétée, par rapport à l'Allemagne entre 1918 et 1939, puis depuis 1991 avec la Russie dont elle partie intégrante après 1945. La symétrie s'interprète par rapport aux enjeux de la continuité terrestre de transit, ainsi que du renforcement des liaisons maritimes. Dans les deux cas, le pouvoir central a doté ces territoires d'investissements matériels (infrastructure notamment) en leur réservant une place importante dans leurs discours politiques respectifs. L'axe de symétrie qui est ici essentiellement temporel invite ainsi à l'analyse comparative des deux situations territoriales soulignant bien l'analogie des situations géographiques.

10 Si l'appartenance successive d'un espace à deux systèmes politiques passe par une rupture complète comme c'est le cas de Kaliningrad où le pouvoir soviétique a éradiqué toute trace du passé allemand, la coexistence simultanée d'éléments faisant référence à deux systèmes étatiques peut conduire à une situation de dualité. Plusieurs stratégies peuvent alors être suivies : celle du déni, de la contradiction ou encore de la réinterprétation ou du détournement. L'analyse du cas strasbourgeois souligne les éléments urbains d'une dualité construite par la frontière et ses déplacements à travers l'analyse de trois lieux emblématiques de la ville. L'évolution géopolitique contemporaine permet aujourd'hui de valoriser et de refonder dans une perspective européenne cette double appartenance naguère problématique. L'article entend 
montrer comment cette opportunité ouvre de nouvelles perspectives au projet urbain dans une perspective de développement assumé entre France et Allemagne.

Philippe Martin avance enfin rien moins que l'exigeante proposition d'une théorie unificatrice de la géographie fondée sur le renouvellement même du concept de symétrie. Au-delà de la seule apparence immédiate, il tente d'en définir le concept de manière radicale. Il s'appuie pour ce faire sur les acquis contemporains des mathématiques, où la notion de symétrie se définit comme une transformation de l'objet avec conservation de forme, que ce soit par translation, rotation, miroir ou dilatation. Il intègre ainsi la dimension de l'échelle, jusque là peu abordée, bien qu'au cœur de l'approche géographique. La symétrie n'est plus alors un phénomène second, mais une notion fondamentale dans l'intelligibilité de la Nature. Dans son texte, l'auteur propose une lecture critique de l'approche euclidienne par trop réductrice, qu'il applique à l'analyse de la démarche chorématique. Pour lui, l'interprétation géographique de la réalité relève bien d'expressions non euclidiennes de la symétrie, auxquelles il convient d'associer les fractales. Tout en rappelant la nécessaire rupture avec l'héritage antique de lecture de formes géométriques simples et immédiatement perceptibles, l'entreprise n'est pas toutefois sans renouer avec la puissance théorique même de la démarche grecque : celle d'une réalité finalement géométrisable, bien que selon des expressions plus complexes.

\section{BIBLIOGRAPHIE}

BERGEVIN J. (1992). - Déterminisme et géographie. Hérodote, Strabon, Albert le Grand et Sébastien Münster, Presses de l'Université de Laval, pp. 31-35.

BRoC N. (1980) - « De l'Antichtone à l'Antarctique », in Cartes et figures de la Terre, Centre Georges Pompidou, pp. 136-149.

CLOSE F. (?) - Asymétrie : la beauté du diable. Où se cache la symétrie de l'Univers ? EDP Sciences.

Colloque « Symétrie, asymétrie, dissymétrie », ENS Lyon, 3 et 4 juin 2004.

SIVARDIÈRE J. (2004) - Description de la symétrie. Des groupes de symétrie aux structures fractales, EDP Sciences, $552 \mathrm{p}$.

\section{NOTES}

1. Dictionnaire grec-français d'Antoine Bailly indique pour "symmetria » $: 1$; réduction à une commune mesure, juste proportion / symétrie, proportion. Dans le Trésor de la Langue française, la symétrie est définie dans son sens ancien comme « un rapport harmonieux de grandeur, de forme, de position que les différentes parties d'un ensemble ont entre elles et avec leur tout ». 


\section{AUTEUR}

ANTOINE BEYER

Université Paris 4, Institut de géographie, 191 rue St Jacques, 75005 Paris, antoinebeyer@yahoo.fr 